\title{
Prevalence of canine Babesia and Ehrlichia co-infection and the predictive value of haematology
}

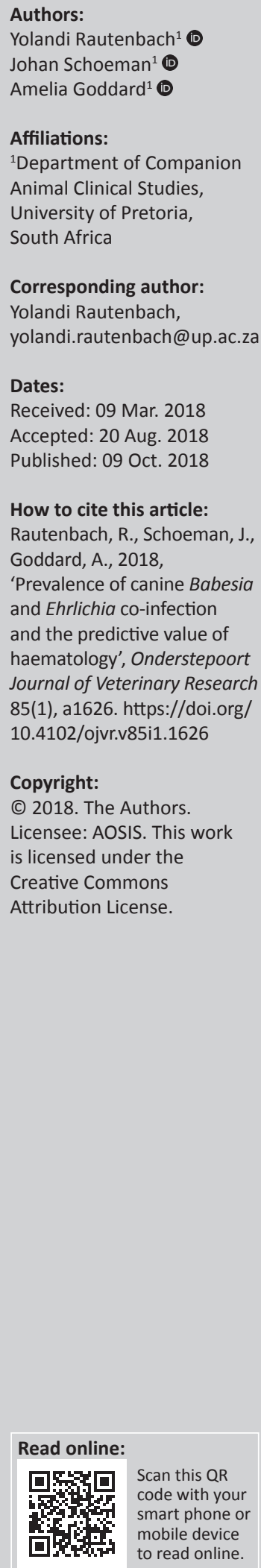

Canine babesiosis and ehrlichiosis are important tick-borne infections in South Africa. Many South African general veterinary practitioners perceive co-infection with Ehrlichia spp. as a common occurrence in dogs with babesiosis. Studies about the prevalence of coinfection in South African dogs are lacking. This retrospective study aimed to determine the prevalence of Ehrlichia co-infection in dogs with babesiosis. Additionally, the predicative value of specific haematological variables for co-infection was evaluated. The study population consisted of 205 dogs diagnosed with canine babesiosis presented to the Onderstepoort Veterinary Academic Hospital (OVAH) in 2006 and between 2011 and 2013. The Babesia-infected dogs were grouped based on presence or absence of an Ehrlichia spp. co-infection. Ehrlichia spp. co-infection was confirmed using polymerase chain reaction. Positive and negative predictive values (PPVs and NPVs) of leukopenia or thrombocytopenia for co-infection were also calculated. The prevalence of Babesia spp. and Ehrlichia spp. co-infection in this cohort of dogs was $2 \%$. In the babesiosis dogs, the PPV of leukopenia for co-infection with Ehrlichia spp. was 1.3\%, and the NPV 97.4\%. Similarly, the PPV and NPVs of thrombocytopenia for co-infection were $2.1 \%$ and $100 \%$, respectively. Co-infection with Ehrlichia spp. was a rare occurrence in dogs with babesiosis presented to the OVAH. Normal leukocyte or platelet counts confidently ruled out the presence of concurrent ehrlichiosis in this cohort of dogs. However, the diagnosis of Ehrlichia co-infection based on the presence of thrombocytopenia or leukopenia would have been associated with false positive results in more than $97.4 \%$ of cases.

\section{Introduction}

Canine babesiosis and ehrlichiosis are important tick-borne infections in South Africa, resulting in severe clinical disease (Collett 2000; Rautenbach, Boomker \& De Villiers 1991; Van Heerden 1982). In South Africa, canine babesiosis is predominantly caused by the virulent Babesia rossi sp. (Matjila et al. 2008). Although several Ehrlichia spp. are able to cause natural disease in dogs, only E. canis and E. ruminantium occur in southern Africa (Kelly 2000; Neer et al. 2002).

Severe thrombocytopenia is a common finding in canine babesiosis (Kettner, Reyers \& Miller 2003; Scheepers et al. 2011), as is leukopenia (Scheepers et al. 2011). Similarly in canine ehrlichiosis, thrombocytopenia and leukopenia are common haematological abnormalities in both the acute and chronic phase of the infection (Kelly 2000; Shipov et al. 2008). Despite the presence of severe thrombocytopenia, clinical bleeding is uncommon in canine babesiosis because of marked platelet activation and marked hyperfibrinogenaemia (Goddard et al. 2015b; Liebenberg et al. 2013); however, excessive bleeding is seen in cases with concomitant Ehrlichia spp. infections (Van Heerden, Reyers \& Stewart 1983), most likely secondary to the thrombocytopenia and thrombocytopathia reported in this disease (Neer 1998).

In endemic areas, infection with multiple tick-borne pathogens is possible in individual animals, especially secondary to a heavy tick infestation (Shaw et al. 2001). A single tick species can act as a vector for multiple pathogens and simultaneous infection with different organisms is possible (Schouls et al. 1999; Shaw et al. 2001). In South Africa, a 19\% infection rate of E. canis was noted in Rhipicephalus sanguineus ticks (Mtshali et al. 2017), but to the authors' knowledge no reports are available on the infection rate of B. rossi in Haemaphysalis elliptica ticks.

Studies about the prevalence of Babesia and Ehrlichia co-infections in dogs in South African are lacking. The majority of veterinary practitioners in South Africa have reported diagnosing canine ehrlichiosis in their practices and more than half consider it an occasional to common co-infection in dogs with babesiosis (Collett 2000). Approximately one-quarter of these practitioners based 
their diagnosis of concurrent Ehrlichia spp. infection on the presence of normal white cell count or leukopenia, while half of the clinicians based their diagnosis on the presence of a thrombocytopenia (Collett 2000).

In addition, a considerable proportion of practitioners are inclined to treat for Ehrlichia spp. co-infection in dogs with babesiosis, without the former being definitively confirmed (Collett 2000). Tetracycline, chloramphenicol, imidocarb dipropionate and amicarbalide are effective in the treatment of ehrlichiosis (Neer 1998). However, doxycycline is considered the drug of choice and a 4 -week oral course is recommended based on the current consensus statement of the Infectious Disease Study Group of the American College of Veterinary Internal Medicine (Eddlestone et al. 2007; Neer et al. 2002). Currently, there are conflicting results about the efficacy of doxycycline in clearing E. canis from the blood and tissues of infected dogs (Eddlestone et al. 2007; Harrus et al. 1998; Iqbal \& Rikihisa 1994; McClure et al. 2010), raising questions about the dosage and duration of doxycycline treatment and the development of potential drug resistance.

The first objective of this study was to determine the prevalence of Ehrlichia spp. co-infection in dogs with babesiosis presented to the Onderstepoort Veterinary Academic Hospital (OVAH). The second objective was to determine the predictive value of thrombocytopenia or leukopenia for co-infection with Ehrlichia spp. in dogs with babesiosis. We hypothesised that the prevalence of coinfection would be low in dogs presented with babesiosis and, additionally, that the presence of leukopenia or thrombocytopenia would not be predictive of concurrent Ehrlichia infection.

\section{Materials and methods}

This retrospective study included the review of medical records of client-owned dogs naturally infected with canine Babesia spp., from two previously performed prospective clinical research studies. The dogs were presented for veterinary care to the OVAH, Gauteng, South Africa, between January and March 2006 (Study 1) and from October 2011 to April 2013 (Study 2).

The research protocols were approved by the University of Pretoria's Animal Ethics Committee (V070-05 and V055-11, respectively). Several studies originating from these two cohorts of dogs have been published (Goddard et al. 2015a, 2015b, 2016; Rees \& Schoeman 2008; Schoeman \& Herrtage 2007, 2008).

Infection with Babesia parasites was initially diagnosed by demonstration of intra-erythrocytic trophozoites on stained thin blood smears, and the species was confirmed as B. rossi by polymerase chain reaction (PCR) and reverse line blot (RLB) (Matjila et al. 2004, 2008). All samples were also screened for $B$. vogeli and E. canis. Owner consent was obtained for enrolment of all the dogs in this study.

\section{Animals}

Suitable dogs sampled during the second study were of any breed and either sex, $>12$ weeks of age and weighed $>3 \mathrm{~kg}$, while dogs from the first study did not have weight or age restrictions. Both groups of dogs had a demonstrable parasitaemia on a stained thin peripheral blood smear and clinical signs consistent with clinical babesiosis. Dogs were excluded if any signs of concurrent chronic or inflammatory disease conditions, any obvious infections or wounds, or any signs of trauma were present. Vaccination, drug therapy (drugs affecting platelet function or serum glucose concentrations, calcium-containing preparations, catecholamines or other adrenergic drugs, insulin therapy) or any unrelated metabolic illness four weeks prior to presentation were also reasons for exclusion.

\section{Sample collection and analyses}

Peripheral venous blood was collected at presentation prior to any treatment. Blood samples were collected from the jugular vein from each dog with a 21-gauge needle by careful venepuncture with minimal stasis. The blood samples were collected into serum and ethylenediaminetetraacetic acid (EDTA) Vacutainer plastic tubes (Becton Dickinson [BD] Biosciences, New Jersey). The EDTA sample was used to perform a complete blood count (CBC), PCR and RLB assays. Because of the prolonged period between the two study populations, CBC data from two different automated cell counters were available: ADVIA 2120 (Siemens, Munich, Germany) and Cell-Dyn 3700 (Abbott Diagnostics, Santa Clara, CA, United States).

\section{Deoxyribonucleic acid extraction and polymerase chain reaction}

Deoxyribonucleic acid (DNA) was extracted from $200 \mu \mathrm{L}$ EDTA-anticoagulated whole blood using a blood and tissue extraction kit (QIAmp blood and tissue extraction kit, Qiagen, Venlo, Netherlands) according to the manufacturer's instructions. Molecular diagnosis of $B$. rossi and exclusion of other Babesia, Theileria, Ehrlichia and Anaplasma spp. were performed using PCR and RLB. Polymerase chain reaction was conducted with a set of primers that amplified a 460-540 base pair fragment of the 18S SSU rRNA spanning the V4 region, a region conserved for Babesia and Theileria. The Ehrlichia PCR amplified the V1 hypervariable region of the 16S SSU rRNA. The membrane used for RLB included probes for B. vogeli, B. rossi, B. canis and E. canis (Matjila et al. 2008).

\section{Statistical analysis}

The Babesia-infected dogs were divided into groups, based on presence or absence of Ehrlichia spp. co-infection. Descriptive statistics were performed using a commercial software package (SPSS Statistics 23.0 ${ }^{\circledR}$ Software; SPSS Inc, StataCorp, College Station, TX, United States). The prevalence of Ehrlichia spp. co-infection in babesiosis dogs was defined as the percentage of babesiosis dogs that tested 
positive on PCR for Ehrlichia spp. The respective positive predictive values (PPVs) of leukopenia and thrombocytopenia for co-infection with Ehrlichia spp. were calculated. $\mathrm{PPV}=\mathrm{TP} /(\mathrm{TP}+\mathrm{FP}) \times 100$, where $\mathrm{TP}$ represents the true positives (Babesia-infected dogs with leukopenia or thrombocytopenia co-infected with Ehrlichia spp.) and FP represents the false positives (Babesia-infected dogs with leukopenia or thrombocytopenia without Ehrlichia spp. co-infection). The respective negative predictive values (NPVs) of a normal leukocyte count and platelet count for the exclusion of co-infection with Ehrlichia spp. were also determined. NPV $=\mathrm{TN} /(\mathrm{TN}+\mathrm{FN}) \times 100$, where $\mathrm{TN}$ represents the true negatives (Babesia-infected dogs with normal leukocyte counts or platelet counts without Ehrlichia spp. co-infection) and FN represents false negatives (Babesiainfected dogs with normal leukocyte counts or platelet counts with Ehrlichia spp. co-infection). Thrombocytopenia was defined as a platelet count $<200 \times 10^{9} / \mathrm{L}$ (populationbased reference interval [RI]: 200-500 $\times 10^{9} / \mathrm{L}$ ) and a leukopenia was defined as a leukocyte count $<6 \times 10^{9} / \mathrm{L}$ (RI: $6-15 \times 10^{9} / \mathrm{L}$ ).

\section{Results}

\section{Study population characteristics}

Of the 205 dogs sampled that were naturally infected with Babesia spp., only 198 dogs were included in the study. Two dogs were excluded because of negative PCR results for any infectious agents and six dogs did not have any PCR results available for analysis. The median age (range) and weight (range) of all the infected dogs were 18 months (9-36) and $18.2 \mathrm{~kg}$ (8.5-28.5) (Table 1), respectively, and included 127 male and 71 female dogs. The most prevalent breeds were mixed-breed dogs (29\%), followed by Boerboels (12\%) and Jack Russell terriers (8\%). The remaining breeds each represented less than $5 \%$ of the population. The median age (range) and weight (range) of the Babesia-infected dogs were 18 months (9.0-36.5) and $18.3 \mathrm{~kg}$ (8.6-28.5), respectively. The median age (range) and weight (range) of the co-infected dogs were 12 months (6-18) and $6.7 \mathrm{~kg}(4.2-23.9)$, respectively (Table 1).

\section{Co-infection prevalence}

Based on PCR results, 191 of the dogs were solely infected with B. rossi (96\%), four dogs were infected with B. vogeli (2\%), of which two were co-infected with E. canis (1\%), one dog was co-infected with both B. rossi and B. vogeli $(0.5 \%)$ and two dogs infected with $B$. rossi were co-infected with E. canis and E. ruminantium (1\%). The prevalence of Babesia spp. and Ehrlichia spp. co-infection in this cohort of dogs was $2 \%$.

\section{Predictive value of haematology results}

The median leukocyte count (range) and platelet count (range) of all the infected dogs were $6.7 \times 10^{9} / \mathrm{L}(4.8-11.90)$ and $17 \times 10^{9} / \mathrm{L}(4-49)$, respectively. In the Babesia-infected dogs, the median leukocyte count (range) was $6.67 \times 10^{9} / \mathrm{L}$ (4.8-11.90) and the median platelet count (range) was $16 \times 10^{9} / \mathrm{L}(4-48)$. The median leukocyte count (range) in coinfected dogs was $8.86 \times 10^{9} / \mathrm{L}(3.88-11.21)$, and the platelet count (range) was $58 \times 10^{9} / \mathrm{L}(31-100)$ (Table 1$)$.

The PPV of leukopenia for co-infection with Ehrlichia spp. in babesiosis dogs was $1.3 \%$, while the NPV was $97.4 \%$ (Table 2). The PPV and NPV of thrombocytopenia for coinfection with Ehrlichia spp. in babesiosis dogs was 2.1\% and $100 \%$, respectively (Table 3 ).

TABLE 1: Population characteristics, leukocyte count and platelet count for dogs with Babesia spp. and Ehrlichia spp. infection.

\begin{tabular}{|c|c|c|c|c|c|c|c|c|c|}
\hline \multirow[t]{2}{*}{ Variable } & \multicolumn{3}{|c|}{ All infected dogs } & \multicolumn{3}{|c|}{ Babesia-infected only } & \multicolumn{3}{|c|}{ Babesia spp. and Ehrlichia spp. co-infection } \\
\hline & Mean \pm SD & Median & IQR & Mean \pm SD & Median & IQR & Mean \pm SD & Median & IQR \\
\hline Age (months) & $30.67 \pm 31.83$ & 18.00 & $9.00-36.00$ & $30.96 \pm 32.00$ & 18.00 & $9.00-36.50$ & $12.00 \pm 6.00$ & 12.00 & $6.00-18.00$ \\
\hline Weight (kg) & $19.88 \pm 13.49$ & 18.20 & $8.50-28.50$ & $20.10 \pm 13.50$ & 18.30 & $8.60-28.50$ & $11.60 \pm 11.77$ & 6.70 & $4.20-23.90$ \\
\hline Leukocyte count $\left(\times 10^{9} / \mathrm{L}\right)$ & $9.28 \pm 6.69$ & 6.70 & $4.80-11.90$ & $9.30 \pm 6.74$ & 6.67 & $4.80-11.90$ & $7.98 \pm 3.92$ & 8.86 & $3.88-11.21$ \\
\hline Platelet count $\left(\times 10^{9} / \mathrm{L}\right)$ & $36 \pm 62$ & 17.00 & $4.00-49.00$ & $35.00 \pm 62.00$ & 16.00 & $4.00-48.00$ & $63.00 \pm 37.00$ & 58.00 & $31.00-100.00$ \\
\hline
\end{tabular}

SD, standard deviation; IQR, interquartile range.

TABLE 2: Contingency table illustrating the relationship between infection status and leukocyte count.

\begin{tabular}{|c|c|c|c|c|c|c|}
\hline \multirow[t]{3}{*}{ Variable } & \multicolumn{4}{|c|}{ Infection status } & \multicolumn{2}{|c|}{ Total } \\
\hline & \multicolumn{2}{|c|}{ Babesia spp. and Ehrlichia spp. co-infection } & \multicolumn{2}{|c|}{ Babesia-infected only } & \multirow[b]{2}{*}{ Number } & \multirow[b]{2}{*}{$\%$} \\
\hline & Number & $\%$ & Number & $\%$ & & \\
\hline Leukopenia & 1 & 1.3 & 78 & 98.7 & 79 & 100.0 \\
\hline Normal leukocyte count & 3 & 2.6 & 113 & 97.4 & 116 & 100.0 \\
\hline Total & 4 & 2.1 & 191 & 97.9 & 195 & 100.0 \\
\hline
\end{tabular}

TABLE 3: Contingency table illustrating the relationship between infection status and platelet count.

\begin{tabular}{|c|c|c|c|c|c|c|}
\hline \multirow[t]{3}{*}{ Variable } & \multicolumn{4}{|c|}{ Infection status } & \multirow{2}{*}{\multicolumn{2}{|c|}{ Total }} \\
\hline & \multicolumn{2}{|c|}{ Babesia spp. and Ehrlichia spp. co-infection } & \multicolumn{2}{|c|}{ Babesia-infected only } & & \\
\hline & Number & $\%$ & Number & $\%$ & Number & $\%$ \\
\hline Thrombocytopenia & 4 & 2.1 & 189 & 97.9 & 193 & 100.0 \\
\hline Normal platelet count & 0 & 0.0 & 2 & 100.0 & 2 & 100.0 \\
\hline Total & 4 & 2.1 & 191 & 97.9 & 195 & 100.0 \\
\hline
\end{tabular}




\section{Discussion}

Published data on the prevalence of co-infection with multiple tick-borne pathogens in South African dogs are very limited. Our study showed that the prevalence of concurrent infections with Babesia and Ehrlichia spp. in South African dogs is very low. Our results concur with those of a previous molecular survey conducted on 1138 blood specimens collected from domestic dogs in South Africa between 2000 and 2006, which reported a $2 \%$ prevalence of $B$. rossi and E. canis co-infection (Matjila et al. 2008). Interestingly, the survey reported that $B$. rossi and E. canis co-infections were found in all of the sampled areas except for the Free State and Eastern Cape provinces (Matjila et al. 2008). Similar to our findings, $1.5 \%$ of dogs were concurrently infected with B. rossi and E. canis, $1.3 \%$ of the dogs had a B. vogeli and E. canis co-infection and only one dog had a concurrent $B$. rossi and B. vogeli infection in the subset of survey samples collected from the OVAH (Matjila et al. 2008).

Our results confirmed that $B$. rossi is the most common cause of babesiosis in dogs presented to the OVAH (Matjila et al. 2008). This correlates with the high percentage of Babesia-infected dogs presented to the OVAH that are infested with H. elliptica, the tick vector for B. rossi (Horak 1995; Matjila et al. 2008). The B. vogeli and E. canis coinfection can be explained by the fact that $R$. sanguineus is the vector for both of these pathogens (Groves et al. 1975; Uilenberg et al. 1989). The mixed infections with B. rossi and E. canis or B. vogeli is most likely because of the overlapping distribution pattern of $R$. sanguineus and H. elliptica and the fact that these vectors commonly infest the same host (Horak 1995; Matjila et al. 2008).

Two of the dogs in our study were co-infected with both E. canis and E. ruminantium. Ehrlichia ruminantium causes heartwater or cowdriosis in all domestic and some wild ruminants and is transmitted by ticks of the genus Amblyomma (Allsopp 2010). The main vector of heartwater in southern Africa is A. hebraeum (Allsopp 2010). Currently, eight different srRNA genotypes of E. ruminantium are known, of which only the Pretoria North genotype has been identified in dogs (Allsopp 2010). This genotype was isolated in South African dogs that presented with clinical symptoms of ehrlichiosis but that tested negative using a PCR assay specific for North American E. canis (Allsopp \& Allsopp 2001). The E. ruminantium organism infecting dogs has not yet been isolated in culture (Allsopp 2010; Allsopp \& Allsopp 2001), thus prohibiting further investigation into its pathogenicity, host specificity and the identification of its vector. Recently, a low number of $A$. hebraeum ticks were found in a South African cohort of dogs and cats (Mtshali et al. 2017). These ticks were negative for the presence of E. canis DNA (Mtshali et al. 2017), but the samples were not screened for E. ruminantium DNA.

Positive predictive value and NPV are utilised for assessing the probability of an accurate diagnosis (Petrie \& Watson 2013). There is an interdependence between prevalence, sensitivity, specificity and predictive values (Hernaez \& Thrift 2017). Prevalence can also be interpreted as the probability that the disease is present before the test is performed (pretest probability) and affects predictive values (Petrie \& Watson 2013). When disease prevalence is low, the NPV is higher and consequently the PPV lower (Hernaez \& Thrift 2017; Petrie \& Watson 2013). Thus, in a low prevalence setting, a negative result confidently excludes the presence of disease (Hernaez \& Thrift 2017; Petrie \& Watson 2013). The opposite is true when the prevalence of the disease increases (Petrie \& Watson 2013). The predictive value of the presence of leukopenia or thrombocytopenia for concurrent ehrlichiosis in our population of dogs with babesiosis was very low, $1.3 \%$ and $2.1 \%$, respectively. In contrast, the predictive value of normal leukocyte count or platelet count for the absence of concurrent ehrlichiosis was very high, $97.4 \%$ and $100 \%$, respectively. Therefore, in light of the low prevalence of co-infection in this population of dogs with babesiosis, a negative test result (i.e. normal leukocyte count or normal platelet count) confidently rules out the presence of concurrent ehrlichiosis. Furthermore, a positive test result (i.e. leukopenia or thrombocytopenia) may result in incorrect diagnosis of co-infection, leading to an over-diagnosis of concurrent ehrlichiosis in Babesia-infected dogs.

The median age of the population of infected dogs in this study was 18 months, which is consistent with previous reports that the majority of dogs with babesiosis are young (Jacobson 2006; Mellanby et al. 2011). More male than female dogs were presented with babesiosis, $64 \%$ compared to $36 \%$, mirroring previous reports (Jacobson 2006). The most common breeds in this study were mixed-breed, followed by Boerboel and Jack Russell terrier. These findings are similar to the reported literature, with mixed-breed dogs most commonly presented for babesiosis while toy breed dogs appear to be less likely to be presented for babesiosis (Jacobson 2006; Mellanby et al. 2011).

A limitation of this study is the fact that the haematology was performed on two different haematology analysers and therefore the results are not comparable. However, the population-based RIs remained unchanged over the study period; therefore, the classification of a leukopenia or thrombocytopenia remained consistent. A further limitation regarding the reported prevalence of Ehrlichia co-infection in South African dogs with babesiosis is that the findings of this study are only applicable to the geographical area of this cohort of dogs. The prevalence might be higher in areas where regular tick prevention is not practised or in areas with a higher concentration of tick vectors. In light of the retrospective nature of this study, the inference was made that the analysed data were unbiased; however, some babesiosis cases with concurrent ehrlichiosis could have inadvertently been excluded. Therefore, the results reported here should be confirmed with a prospective study.

\section{Conclusion}

It can thus be concluded from these results that concurrent ehrlichiosis is uncommon in dogs with babesiosis presented 
to the OVAH, situated north of Pretoria in Gauteng. Although thrombocytopenia is a common finding in dogs with babesiosis, its PPV for co-infection is very low, similar to the presence of leukopenia. Therefore, the diagnosis of Ehrlichia co-infection based on thrombocytopenia or leukopenia is associated with many FP results.

\section{Acknowledgements Competing interests}

The authors declare that they have no financial or personal relationships that may have inappropriately influenced them in writing this article.

\section{Authors' contributions}

Y.R. participated in the planning and design of the study, analysed, evaluated and interpreted the data, and compiled and structured the manuscript. J.S. was involved with the study design and data collection and assisted with the data analysis and interpretation. A.G. conceived the original study idea, was involved in the study design and data collection and assisted with the data analysis and interpretation. All authors contributed to the main text.

\section{References}

Allsopp, B.A., 2010, 'Natural history of Ehrlichia ruminantium', Veterinary Parasitology 167, 123-135. https://doi.org/10.1016/j.vetpar.2009.09.014

Allsopp, M.T. \& Allsopp, B.A., 2001, 'Novel Ehrlichia genotype detected in dogs in South Africa', Journal of Clinical Microbiology 39, 4204-4207. https://doi. org/10.1128/JCM.39.11.4204-4207.2001

Collett, M.G., 2000, 'Survey of canine babesiosis in South Africa', Journal of the South African Veterinary Association 71, 180-186. https://doi.org/10.4102/ jsava.v71i3.710

Eddlestone, S.M., Diniz, P.P., Neer, T.M., Gaunt, S.D., Corstvet, R., Cho, D. et al., 2007 'Doxycycline clearance of experimentally induced chronic Ehrlichia canis infection in dogs', Journal of Veterinary Internal Medicine 21, 1237-1242. https://doi. in dogs', Journal of Veterinary Internal
org/10.1111/j.1939-1676.2007.tb01944.x

Goddard, A., Leisewitz, A.L., Kjelgaard-Hansen, M., Kristensen, A.T. \& Schoeman, J.P., 2016, 'Excessive pro-inflammatory serum cytokine concentrations in virulent canine babesiosis', PLoS One 11, e0150113. https://doi.org/10.1371/journal.pone. 0150113

Goddard, A., Leisewitz, A.L., Kristensen, A.T. \& Schoeman, J.P., 2015a, 'Platelet activation and platelet-leukocyte interaction in dogs naturally infected with Babesia rossi', Veterinary Journal 205, 387-392. https://doi.org/10.1016/j.tvjl. 2015.05.008

Goddard, A., Leisewitz, A.L., Kristensen, A.T. \& Schoeman, J.P., 2015b, 'Platelet indices in dogs with Babesia rossi infection', Veterinary Clinical Pathololgy 44, 493-497. https://doi.org/10.1111/vcp.12306

Groves, M.G., Dennis, G.L., Amyx, H.L. \& Huxsoll, D.L., 1975, 'Transmission of Ehrlichia canis to dogs by ticks (Rhipicephalus sanguineus)', American Journal of Veterinary Research 36, 937-940.

Harrus, S., Waner, T., Aizenberg, I. \& Bark, H., 1998, 'Therapeutic effect of doxycycline in experimental subclinical canine monocytic ehrlichiosis: Evaluation of a 6-week course', Journal of Clinical Microbiology 36, 2140-2142.

Hernaez, R. \& Thrift, A.P., 2017, 'High negative predictive value, low prevalence, and spectrum effect: Caution in the interpretation', Clinical Gastroenterology and Hepatology 15, 1355-1358. https://doi.org/10.1016/j.cgh.2017.05.002

Horak, I.G., 1995, 'Ixodid ticks collected at the Faculty of Veterinary Science, Onderstepoort, from dogs diagnosed with Babesia canis infection', Journal of the South African Veterinary Association 66, 170-171.

Iqbal, Z. \& Rikihisa, Y., 1994, 'Reisolation of Ehrlichia canis from blood and tissues of dogs after doxycycline treatment', Journal of Clinical Microbiology 32, 1644-1649.
Jacobson, L.S., 2006, 'The South African form of severe and complicated canine babesiosis: Clinical advances 1994-2004', Veterinary Parasitology 138, 126-139. https://doi.org/10.1016/j.vetpar.2006.01.047

Kelly, P.J., 2000, 'Canine ehrlichioses: An update', Journal of the South African Veterinary Association 71, 77-86. https://doi.org/10.4102/jsava.v71i2.684

Kettner, F., Reyers, F. \& Miller, D., 2003, 'Thrombocytopenia in canine babesiosis and its clinical usefulness', Journal of the South African Veterinary Association 74 63-68. https://doi.org/10.4102/jsava.v74i3.512

Liebenberg, C., Goddard, A., Wiinberg, B., Kjelgaard-Hansen, M., Van der Merwe, L.L., Thompson, P.N. et al., 2013, 'Hemostatic abnormalities in uncomplicated babesiosis (Babesia rossi) in dogs', Journal of Veterinary Internal Medicine 27 150-156. https://doi.org/10.1111/jvim.12016

Matjila, P.T., Leisewitz, A.L., Jongejan, F. \& Penzhorn, B.L., 2008, 'Molecular detection of tick-borne protozoal and ehrlichial infections in domestic dogs in South Africa', Veterinary Parasitology 155, 152-157. https://doi.org/10.1016/j.vetpar.2008. 04.012

Matjila, P.T., Penzhorn, B.L., Bekker, C.P., Nijhof, A.M. \& Jongejan, F., 2004, 'Confirmation of occurrence of Babesia canis vogeli in domestic dogs in South Africa', Veterinary Parasitology 122, 119-125. https://doi.org/10.1016/j.vetpar.2004.03.019

McClure, J.C., Crothers, M.L., Schaefer, J.J., Stanley, P.D., Needham, G.R., Ewing, S.A et al., 2010, 'Efficacy of a doxycycline treatment regimen initiated during et al., 2010, 'Efficacy of a doxycycline treatment regimen initiated during
three different phases of experimental ehrlichiosis', Antimicrobial Agents and Chemotherapy 54, 5012-5020. https://doi.org/10.1128/AAC.01622-09

Mellanby, R.J., Handel, I.G., Clements, D.N., Bronsvoort, B.M., Lengeling, A. \& Schoeman, J.P., 2011, 'Breed and sex risk factors for canine babesiosis in South Africa', Journal of Veterinary Internal Medicine 25, 1186-1189. https://doi.org/ 10.1111/j.1939-1676.2011.00779.x

Mtshali, K., Nakao, R., Sugimoto, C. \& Thekisoe, O., 2017, 'Occurrence of Coxiella burnetii, Ehrlichia canis, Rickettsia species and Anaplasma phagocytophilum-like bacterium in ticks collected from dogs and cats in South Africa', Journal of the South African Veterinary Association 88, e1-e6. https://doi.org/10.4102/jsava. South African
v88i0.1390

Neer, T.M., 1998, 'Canine monocytic and granulocytic ehrlichiosis', in Greene, C.E. (ed.), Infectious diseases of the dog and cat, 2nd edn., pp. 139-147, WB Saunders, Philadelphia, PA.

Neer, T.M., Breitschwerdt, E.B., Greene, R.T. \& Lappin, M.R., 2002, 'Consensus statement on ehrlichial disease of small animals from the infectious disease study group of the ACVIM. American College of Veterinary Internal Medicine', Journal of Veterinary Internal Medicine 16, 309-315.

Petrie, A. \& Watson, P.F., 2013, Statistics for veterinary and animal science, WileyBlackwell, Chichester.

Rautenbach, G.H., Boomker, J. \& De Villiers, I.L., 1991, 'A descriptive study of the canine population in a rural town in southern Africa', Journal of the South African Veterinary Association 62, 158-162.

Rees, P. \& Schoeman, J.P., 2008, 'Plasma insulin concentrations in hypoglycaemic dogs with Babesia canis rossi infection', Veterinary Parasitology 152, 60-66. https:// doi.org/10.1016/j.vetpar.2007.11.017

Scheepers, E., Leisewitz, A.L., Thompson, P.N. \& Christopher, M.M., 2011, 'Serial haematology results in transfused and non-transfused dogs naturally infected with Babesia rossi', Journal of the South African Veterinary Association 82 with Babesia rossi', Journal of the South African
136-143. https://doi.org/10.4102/jsava.v82i3.51

Schoeman, J.P. \& Herrtage, M.E., 2007, 'The response of the pituitary-adrenal and pituitary-thyroidal axes to the plasma glucose perturbations in Babesia canis rossi babesiosis', Journal of the South African Veterinary Association 78, 215-220. https://doi.org/10.4102/jsava.v78i4.326

Schoeman, J.P. \& Herrtage, M.E., 2008, 'Adrenal response to the low dose ACTH stimulation test and the cortisol-to-adrenocorticotrophic hormone ratio in canine babesiosis', Veterinary Parasitology 154, 205-213. https://doi.org/10.1016/j. vetpar.2008.03.023

Schouls, L.M., Van De Pol, I., Rijpkema, S.G. \& Schot, C.S., 1999, 'Detection and identification of Ehrlichia, Borrelia burgdorferi sensu lato, and Bartonella species in Dutch Ixodes ricinus ticks', Journal of Clinical Microbiology 37, 2215-2222.

Shaw, S.E., Day, M.J., Birtles, R.J. \& Breitschwerdt, E.B., 2001, 'Tick-borne infectious diseases of dogs', Trends Parasitology 17, 74-80. https://doi.org/10.1016/S14714922(00)01856-0

Shipov, A., Klement, E., Reuveni-Tager, L., Waner, T. \& Harrus, S., 2008, 'Prognostic indicators for canine monocytic ehrlichiosis', Veterinary Parasitology 153, 131-138. https://doi.org/10.1016/j.vetpar.2008.01.009

Uilenberg, G., Franssen, F.F., Perie, N.M. \& Spanjer, A.A., 1989, 'Three groups of Babesia canis distinguished and a proposal for nomenclature', Veterinary Quarterly 11, 33-40. https://doi.org/10.1080/01652176.1989.9694194

Van Heerden, J., 1982, 'A retrospective study on 120 natural cases of canine ehrlichiosis', Journal of the South African Veterinary Association 53, 17-22.

Van Heerden, J., Reyers, F. \& Stewart, C.G., 1983, 'Treatment and thrombocyte levels in experimentally induced canine ehrlichiosis and canine babesiosis', Onderstepoort Journal of Veterinary Research 50, 267-270. 